\title{
A survey of the exposure to Ostertagia ostertagi in dairy cow herds in Europe through the measurement of antibodies in milk samples from the bulk tank
}

\author{
A.B. Forbes ${ }^{\mathrm{a}, *}$, J.Vercruysse $^{\mathrm{b}}$, J. Charlier $^{\mathrm{b}}$ \\ ${ }^{a}$ Merial SAS, 29 Avenue Tony Garnier, Lyon 69007, France \\ ${ }^{\mathrm{b}}$ Department of Virology, Parasitology and Immunology, Faculty of Veterinary Medicine, Ghent University, \\ Salisburylaan 133, 9820 Merelbeke, Belgium
}

Received 12 May 2008; received in revised form 19 June 2008; accepted 24 June 2008

\begin{abstract}
Measurement of antibodies to Ostertagia ostertagi in bulk tank milk (BTM) has value as a diagnostic indicator for potential production losses and anthelmintic treatment responses in dairy herds. Most of the recent data on O. ostertagi antibodies in milk have been generated in Belgium and Canada; the purpose of this study was to determine the range of $O$. ostertagi antibody levels in several European countries. BTM samples were collected during the autumn of 2005 and 2006 from a total of 1185 dairy herds from dairy farming regions in Denmark, Germany, Ireland, Italy, the Netherlands, Portugal, Spain and the United Kingdom. Antibody titres to $O$. ostertagi were determined by indirect ELISA and expressed as optical density ratios (ODR). In addition, relationships between ODR and management practices were investigated. For each country the mean ODR and the 25th-75th percentile values were determined. Mean BTM ODR values in herds with access to yards, paddocks and pastures ranged from 0.3 in Italy to 0.6 in Portugal and the UK/Ireland. The BTM ODR values obtained in this study were generally lower than those described in the literature for Belgium, but comparable with those in Canada. Variations between different European countries appeared to reflect different husbandry practices, particularly those related to access to pasture. The association analyses showed correlations between the BTM O. ostertagi ODR, outside access and grazing management, consistent with the publications from Belgium and Canada. When diagnostic values appropriate for different production situations and environments have been further validated, the test will provide an objective, quantitative assessment of the $O$. ostertagi status of a dairy herd and the possible impact this may have on performance and potential responses to anthelmintic treatment. This represents a significant step forward in evidence-based medicine for dairy veterinarians, advisors and farmers.
\end{abstract}

(C) 2008 Elsevier B.V. All rights reserved.

Keywords: Ostertagia ostertagi; ELISA; Bulk milk; Diagnosis; Eprinomectin

\section{Introduction}

Although there is evidence of a significant negative impact of nematode infections on milk production

\footnotetext{
* Corresponding author. Tel.: +33 472726329; fax: +33472723298.

E-mail address: andy.forbes@merial.com (A.B. Forbes).
}

(Gross et al., 1999; Sanchez et al., 2004a), treatment responses to anthelmintics can vary amongst different studies and herds (Kloosterman et al., 1996; Michel et al., 1982; O'Farrell et al., 1986; Ploeger et al., 1990; Ploeger et al., 1989). One possible explanation for the variability in the responses to anthelmintic treatment is that, not only do individual cows in a herd carry worm burdens of different magnitudes, but also that dairy 
herds could be grazing pastures with different levels of exposure to, and hence challenge from, infective larvae of Ostertagia ostertagi (Eysker et al., 2002; Fox et al., 2007).

Neither faecal egg counts nor plasma pepsinogen values have been shown to provide quantitative measures of worm burdens, or their impact, in adult cattle (Eysker and Ploeger, 2000; Vercruysse and Claerebout, 2001). On the other hand, antibodies against crude extracts of $O$. ostertagi antigens, measured by ELISA in serum (Ploeger et al., 1989) and milk (Kloosterman et al., 1993), have been shown to vary quantitatively both amongst individual cows and between herds, raising the possibility of discriminating different levels of infection and exposure to parasites. $O$. ostertagi antibody levels, whether measured in individual sera, milk or bulk milk, were also found to negatively correlate with milk yield (Kloosterman et al., 1993). Subsequent studies confirmed the negative relationship between bulk tank milk (BTM) antibody levels and herd milk yield (Charlier et al., 2005b; Guitian et al., 2000; Sanchez and Dohoo, 2002; Sanchez et al., 2004b).

The potential for the mean herd level of $O$. ostertagi serum antibodies to be used as a predictor of a milk yield response to anthelmintic treatment in adult dairy cows was first described in the Netherlands in cows that were treated with ivermectin in the dry period during housing (Ploeger et al., 1989). The mean herd milk production response to treatment correlated positively $(P<0.05)$ with the mean herd $O$. ostertagi antibody titre measured in the September prior to housing. Another study in the Netherlands, but using bulk milk rather than serum as the substrate for testing, demonstrated an association between the herd titre for $O$. ostertagi antibodies in the bulk milk tank and the treatment response to ivermectin administered in the dry period $>28$ days before calving (Kloosterman et al., 1996). The associations were not significant, possibly because of the relatively small sample size ( 34 farms) and because the animals were not treated during lactation. Subsequent studies using eprinomectin, which was generally administered during lactation (some animals in the study of Charlier et al., 2007b were in late pregnancy), indicated that the magnitude of the increase in milk yield subsequent to treatment was positively related to $O$. ostertagi antibody levels in both individual cows (Sanchez et al., 2005; Sanchez et al., 2002a) and in BTM (Charlier et al., 2007b; Sithole et al., 2005).

All the studies cited have been conducted in a relatively narrow geographic range in Europe (Belgium and the Netherlands) or in Canada, so it was considered important that BTM O. ostertagi antibody levels should be surveyed throughout a number of European countries to determine if the values were comparable to those found in these three countries. If the ranges in BTM $O$. ostertagi antibody values and the associations with various management factors were similar, then extrapolations from the original studies might be reasonably considered.

The objective of this survey was to investigate the $O$. ostertagi status of dairy cow herds in different European countries through measurement of antibody concentration in the bulk milk tank and to compare these results with those already published for Belgium and Canada. Additionally, management and performance data were collected where possible in order to determine if relationships between BTM $O$. ostertagi antibody levels and various management practices and performance indicators were similar.

\section{Materials and methods}

\subsection{Sampling procedure}

BTM samples were collected in the autumn of 2005 and 2006 in eight different European countries: Denmark, Germany, Ireland, Italy, the Netherlands, Portugal, Spain and the United Kingdom. Autumn was chosen in order to limit any differences that might result from seasonal variations (Charlier et al., 2007a) and to determine antibody levels at the end of the grazing season after exposure outside to infection with $O$. ostertagi. Sampling was carried out mainly by practising veterinarians, animal health organisations or through milk recording agencies. The farms were selected by convenience: no specific criteria were imposed for inclusion in the survey, other than that farms should have good records so that management data could be readily collected. In addition, in each country, it was requested that some permanently housed herds with no access to pasture be included in order to establish some base-line data. The target was to obtain BTM samples from a minimum of 100 herds within a country or region.

Samples were collected in a container with a preservative, which was dated and labelled. After collection, samples were initially refrigerated at $4{ }^{\circ} \mathrm{C}$ and then stored at $-20{ }^{\circ} \mathrm{C}$ pending transport to the laboratory. When all the samples from one region had been collected, they were sent with cooling blocks in insulated packaging to arrive within $48 \mathrm{~h}$ at the laboratory of parasitology in Ghent, where all the $O$. ostertagi antibody measurements were conducted. 


\subsection{ELISA protocol}

The $O$. ostertagi antibody levels in milk were determined with an ELISA, as described previously (Charlier et al., 2005c). The test results were expressed as an optical density ratio (ODR).

\subsection{Management information}

Farm management information was collected at each location, generally by veterinarians on site, but, for example in the Netherlands, the data were provided by the farmers themselves by completing on-line or postal forms. The data collected included general background information about the farm, the type of enterprise (dairy \pm beef), herd size (adult cows) and average herd milk production. The milk production data were taken from standard records supplied to the farmer by the milk collection and recording services. In addition, the number and age of young stock (calves were defined as $<1$ year of age) were determined along with details of the parasite control practices used in these animals. Heifers were defined as animals from 1 year of age up to 1st calving; adult cows were defined as adult animals from their first calving onwards and were further subdivided into those that were lactating and those that were not milking, hence dry. The data were subsequently transferred on to a spreadsheet and the responses digitised in order to facilitate statistical analysis.

\subsection{Data analysis}

In order to compare the BTM ODR results from dairy herds amongst countries, the following values were derived from each data set: mean, standard deviation, 25th-75th percentile and minimum and maximum values. Differences in ODR values between countries were tested for statistical significance by one-way ANOVA using Bonferroni's multiple comparison technique with a $5 \%$ overall error rate. The comparative data were restricted to herds that had access to pasture, i.e. excluding herds that were totally confined. The ODR results obtained in this survey were compared with previously reported European data from Belgium (Charlier et al., 2005b; Charlier et al., 2007b) and France (Guiot et al., 2007) and also with Canadian data (Sanchez and Dohoo, 2002; Sanchez et al., 2002a; Sanchez et al., 2002b; Sanchez et al., 2004b; Sanchez et al., 2005; Sithole et al., 2005).

Within each country and depending on the amount of data collected, univariate and/or multivariate regression models were built to investigate associations of management variables of pastured dairy herds with BTM O. ostertagia ODR. If the information was available, herd size and average milk production were included as covariates. Zero-grazing herds were not included in these models. However, based on the Spanish data, a model was built including only zerograzing herds to assess the effect of heifer management on BTM ODR. Associations were considered significant in the univariate analyses if $P \leq 0.01$ and in the multivariate analyses if $P \leq 0.05$.

\section{Results}

\subsection{O. ostertagi BTM ODR per country}

The total number of useable samples for ELISA assay was 1185 and the range per country was from 43 for Ireland to 299 for the Netherlands. The results from samples from the UK and Ireland were combined in the statistical analyses, making a total of seven data sets. The results of the analyses on $O$. ostertagi ODR are presented in Table 1. Comparisons between countries must be viewed with some caution as the inclusion of farms in the survey was not strictly random (see Section 2.1) and the year of sampling differed between 2005 and 2006 The mean BTM ODR in Italy (0.31) was significantly lower than that in all other countries $(P<0.05)$. The other countries segregated into two groups: Portugal, Spain and UK/Ireland and Denmark, Netherlands and Germany: the former three countries had higher means (0.53-0.61) and the latter three were intermediate (0.450.48 ) between these countries and Italy. Comparative BTM ODR value ranges for Belgium and France from the published literature are shown in Table 2. From the four data sets available, comprising results from over 3000 herds, it can be seen that the mean values of the herd $O$. ostertagi ODRs ranged from 0.60 to 0.97 .

\subsection{Association analyses}

The data available, the number of herds in various categories, the criteria and the classification and subdivision of the various criteria varied somewhat amongst the different data sets. Therefore, the associations that were significantly associated with BTM $O$. ostertagi ODR have been grouped according to type and also have been weighted according to whether the relationships were present in more than one region/set of analyses or not; they are shown in Table 3. Regions for which association analyses were possible were Denmark, the Netherlands, Germany, United Kingdom/ 
Table 1

Ostertagia ostertagi BTM ELISA (ODR) profiles in European dairy herds with access to pasture, by country

\begin{tabular}{llllllrr}
\hline Country & $\begin{array}{l}\text { No. of herds } \\
\text { (pastured/total) }\end{array}$ & Sampling time & Mean ODR & $\begin{array}{l}\text { 25th } \\
\text { percentile }\end{array}$ & $\begin{array}{l}\text { 75th } \\
\text { percentile }\end{array}$ & Minimum & Maximum \\
\hline Denmark & $146 / 146$ & Autumn 2005 & 0.48 & 0.26 & 0.67 & 0.04 & 1.23 \\
Germany & $78 / 131$ & Autumn 2006 & 0.48 & 0.29 & 0.65 & 0.01 \\
Italy & $47 / 140$ & Autumn 2006 & 0.31 & 0.13 & 0.48 & -0.09 \\
Netherlands & $243 / 288$ & Autumn 2006 & 0.45 & 0.31 & 0.60 & -0.19 & 1.57 \\
Portugal & $92 / 163$ & Autumn 2006 & 0.61 & 0.54 & 0.71 & -0.06 \\
Spain & $91 / 143$ & Autumn 2006 & 0.53 & 0.27 & 0.81 & -0.10 & 1.00 \\
UK/Ireland & $142 / 174$ & Autumn 2005 & 0.60 & 0.49 & 0.70 & 0.14 \\
\hline
\end{tabular}

Table 2

Ostertagia ostertagi BTM ELISA (ODR) profiles in European dairy herds with access to pasture, from the published literature

\begin{tabular}{lcllllrlrl}
\hline Country & No. of herds & Sampling time & Mean ODR & $\begin{array}{l}\text { 25th } \\
\text { percentile }\end{array}$ & $\begin{array}{l}\text { 75th } \\
\text { percentile }\end{array}$ & Minimum & Maximum & Reference \\
\hline Belgium & 2553 & Spring 2003 & 0.82 & 0.70 & 0.96 & -0.04 & 1.52 & Charlier et al. (2005b) \\
Belgium & 2104 & Autumn 2003 & 0.97 & 0.83 & 1.11 & -0.13 & 1.90 & Charlier et al. (2005b) \\
Belgium & 110 & Autumn 2004 & 0.69 & 0.63 & 0.77 & 0.14 & 1.06 & Charlier et al. (2007b) \\
France & 940 & Spring 2005 & 0.60 & 0.48 & 0.72 & 0.13 & 1.37 & Guiot et al. (2007) \\
\hline
\end{tabular}

Table 3

Significant $(P<0.05)$ associations between herd Ostertagia ostertagi BTM ELISA (ODR) and production/management factors

\begin{tabular}{llll}
\hline Criteria & Associations & Countries $^{*}$ & Literature \\
\hline Access to pasture & No access, low ODR, access high ODR & DE, GE, NL, UK/I, SP & BE, CAN \\
Type of paddock & $\begin{array}{l}\text { Progressive increase in ODR from no access } \\
\text { to exercise paddock to sparsely grassed }\end{array}$ & SP, UK/I & BE, CAN
\end{tabular}

Grazing regime

Month of turnout

Month of housing

Stocking density

Herd size

Milk yield

Anthelmintics given to cows

Organic herds

Access of calves $<12$ months to grass

Dry cows running with young stock

Number of 1st lactation cows

Liver fluke (farmer questionnaire)

Age at first calving

Breed to exercise paddock to sparsely grassed

paddock to fully grassed paddock with

significant herbage intake

ODR increases proportionally to the $\%$ of

time spent grazing each day

ODR increases the earlier the month of turnout

ODR increases the later the month of housing

The higher the stocking density the lower the ODR

ODR decreases with increased herd size

ODR negatively associated with yield

ODR less in treated herds

Associated with higher ODR, but there is

also a confounding effect because of

mandated high $\%$ of time spent

grazing/day in organic systems

In zero-grazed herds with calves with no SP

access to pasture, ODR was lower

If dry cows did not run with young stock, SP

ODR was lower

Negative association with ODR

Positive association between Ostertagia ODR

and reported presence of Fasciola hepatica

Positive association

Holstein herds had higher ODRs compared to

herds comprising Normande or Montbeliard

$\begin{array}{ll}\text { DE, NL, GE, SP } & \text { BE } \\ \text { DE, NL, FR } & \text { BE } \\ \text { NL, UK/I } & \\ \text { SP, UK/I } & \\ \text { DE, GE, NL, SP } & \text { BE } \\ \text { DE, SP, FR } & \text { BE, CAN, NL } \\ \text { FR, UK/I } & \text { CAN } \\ \text { DE } & \\ & \\ \text { SP } & \\ \text { SP } & \\ \text { NL } & \\ \text { NL } & \\ \text { GE } & \text { FR }\end{array}$

* BE: Belgium, CAN: Canada, DE: Denmark, FR: France, GE: Germany, NL: Netherlands, SP: Spain, UK/I: United Kingdom and Ireland. 
Ireland and Spain. The fourth column of Table 3 indicates if a particular association has also been reported in one or more of the published studies from Belgium, Canada or the Netherlands.

The most consistent and well-supported associations are those that relate to access to pasture, with marked differences in BTM ODR between herds that were housed all the time and those that were grazed or exercised outside. An example from the analysis of the data from the Netherlands illustrates the range differences in ODR values between such herds (Fig. 1). In Spain, unexpectedly high ODR values in some zero-grazed herds was explained through a significant association with young animals having access to pasture prior to joining the milking herd. Additionally, the type of outside paddocks and the proportion of time spent there each day were also associated with BTM $O$. ostertagi ODR, as were month of turnout and month of housing. There were also negative associations between BTM ODR and anthelmintic use in cows and herd size. The associations with O. ostertagi ODR that were observed only in single data sets include organic status (positive), presence of liver fluke (positive), age at first calving (positive) and number of first lactation cows (negative).

\section{Discussion}

\subsection{Comparison of BTM ODR values with previously published data}

With the exception of Italy, the mean $O$. ostertagi ODR values in milk from the bulk tank samples

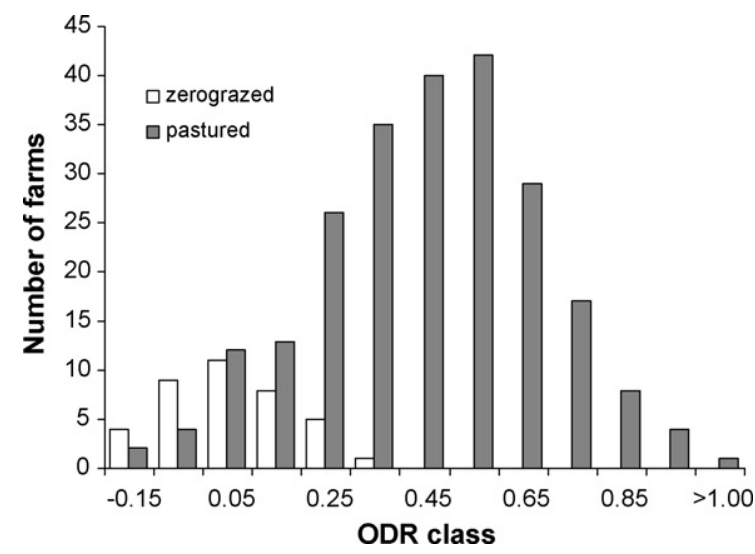

Fig. 1. Distribution of bulk tank milk O. ostertagia ELISA classes between zero-grazed (confined) and pastured herds. Mean ODR of pastured herds is 0.453 (S.D. $=0.227 ; n=233$ ), mean ODR of zerograzed herds is 0.059 (S.D. $=0.119 ; n=38$ ). (H. Ploeger, personal communication). collected in the European survey in 2005/2006 were within the range $0.48-0.60$. For France, the mean value for samples collected in Spring 2005 was of the same order at 0.60. The mean ODRs in the samples taken from herds in Belgium between 2003 and 2006 were consistently higher than in the other European countries: it cannot be determined from this survey if this is a systematic difference based on different management systems, differences in parasite challenge or other local factors. Within the same season and year, it seems unlikely that local weather or climate differences could alone account for the different profiles, because, for example, the values seen in the neighbouring region of the Netherlands differ from those in Belgium. What is apparent for the Belgian figures is that there is some variation between years in the mean herd ODR values which is possibly related to local weather conditions that could affect parasite epidemiology and hence the magnitude of parasite populations (Yazwinski and Tucker, 2006). This possibility is supported by results of bulk milk sampling in the United Kingdom in Autumn 2007 (unpublished data), after a summer with above average rainfall, when ODR results were generally much higher than in the same region in Autumn 2005.

Although not directly comparable because of differences in methodology and timing, the results from Canada are also of interest in the context of the European results. In three studies in dairy herds, in the majority $(>85 \%)$ of which cows had access to pasture during the summer, the mean $O$. ostertagi antibody levels in BTM were 0.69 (OD), 0.54 (ODR) and 0.36 (ODR) (Guitian et al., 2000; Sanchez and Dohoo, 2002; Sanchez et al., 2002a). In a subsequent study, $O$. ostertagi antibodies in the bulk tank were evaluated in confined and semi-confined dairy herds (Sithole et al., 2005). The overall mean $O$. ostertagi ODR in the bulk milk of the 66 herds was 0.41 , with the average in confined herds being 0.38 and that in semi-confined being 0.44 , but these mean values were not significantly different.

Interestingly and surprisingly, the confined herds showed a similar seasonal pattern of BTM ODR values as the semi-confined herds, with peak values under both systems appearing in September. This observation inevitably provokes the question of whether all the herds were in fact totally 'confined' according to the study definition. In the current survey, Spanish herds that were described as 'zero-grazed', but which had higher than expected $O$. ostertagi ODRs, all proved to have grazed their young heifers on pastures, and this fact appeared to explain the apparent discrepancy. It is 
also instructive to consider the results of Charlier et al. (2005a) from Belgium, where it appears that 'zerograzed' herds truly did not have any outside access and to compare the results of different degrees of access to the outside. In that study, the mean ODR values were 0.05 for herds kept in total confinement, 0.75 in those with access to an outside exercise area with little/no grass, 0.94 in herds with access to a small area of pasture and 0.96 for cows with free access to pasture from which they derived a significant proportion of their nutrient intake. It is apparent that even seemingly modest access to the outside in exercise yards or paddocks resulted in cattle being exposed to $O$. ostertagi, manifested by ODR values that approached those of herds with free access to pasture. This finding is unexpected and it challenges some of the assumptions regarding 'high' and 'low' risk farms from a parasitological perspective (MAFF, 1980).

\subsection{Association analyses}

The correlations between bulk milk BTM $O$. ostertagi ODR and various management and production-related parameters have been summarised in Table 3. All but one of the associations that were demonstrated in this European survey in more than one country has also been reported in the literature from Belgium, Canada or both. Thus, the relationships between bulk milk $O$. ostertagi ODR and the main parameters of grazing management and milk production are consistent across geography and time. The only exception to this is month of housing, which is generally related to the length of the grazing season. As housing is determined largely by weather insofar as it affects grass growth and general conditions on the pasture, it is likely to be quite closely synchronised within regions and hence difficult to discriminate amongst different farms. The results of the association analyses in this European survey provide strong support for the information already published on the value of the bulk milk $O$. ostertagi ELISA as an epidemiological and diagnostic tool.

\subsection{Practical implications}

A commercial $O$. ostertagi milk ELISA kit is now available from Svanova ${ }^{\circledR}$ (www.svanova.com) and it is important that guidance is provided for veterinarians and other advisors to farmers regarding the interpretation of results from bulk milk samples. From an economic aspect, one important relationship is that between BTM $O$. ostertagi ODR and milk production: the higher the ODR, the lower the herd's milk production. In pasture-based systems, comparison of daily milk yield from the 25th percentile ODR with those at the 75th percentile ODR, show that cows in herds with the higher ODR value produced on average 0.9-1.25 kg less milk per day (Charlier et al., 2005b; Guitian et al., 2000; Sanchez and Dohoo, 2002). This association was consistent across the various data sets with mean herd $O$. ostertagi ELISA values that varied between 0.54 and 0.97 . It seems reasonable to infer, given the consistency of correlations previously described in Section 4.2, that the negative relationship between BTM ODR and milk yield is valid throughout Europe. Nevertheless, given the variation in mean ODR and percentile values between countries and between years, it may be difficult to compile absolute thresholds or ranges over which effects can be predicted. Similarly, whilst milk ODR has been shown to have value as a guide to the likelihood of an increase in milk yield following eprinomectin treatment, both at the level of the individual cow (Sanchez et al., 2002a; Sanchez et al., 2005) and at the herd level, using BTM samples (Charlier et al., 2007b), absolute ranges and thresholds may be more difficult to establish over wider geography and between years.

A chart has been designed to aid the user of the Svanova kit in the interpretation of the BTM test results (Fig. 2). The level up to which the effect of O. ostertagi on milk yield is considered to be zero is arbitrarily set at 0.5 ODR, based on the 25th percentile ODR values of different countries in the current study and previous studies in Belgium and France. From this inflection point onwards, the relationship between average annual milk yield and Ostertagia ODR is displayed linearly, using data primarily from a Belgian study (Charlier et al., 2005b). Error bars indicate that the magnitude of this relationship can vary somewhat. It is possible that in the future and based on regional epidemiological surveys, the chart may be adapted to local conditions. Like many diagnostic tests, Ostertagia antibody titres in bulk-milk should not be the sole discriminator in the decision-making process regarding estimated losses and potential response to treatment. On-farm, qualitative analysis of management factors can be used to complement the BTM ODR results in terms of assessing losses due to gastrointestinal parasitism.

The decision to treat dairy cows or not also rests on numerous factors other than the BTM results, including the risk of other parasites such as gastro-intestinal nematodes other than $O$. ostertagi, lungworm, mange and lice. In financial terms the ratio of the value of additional milk produced to the cost of treatment needs 


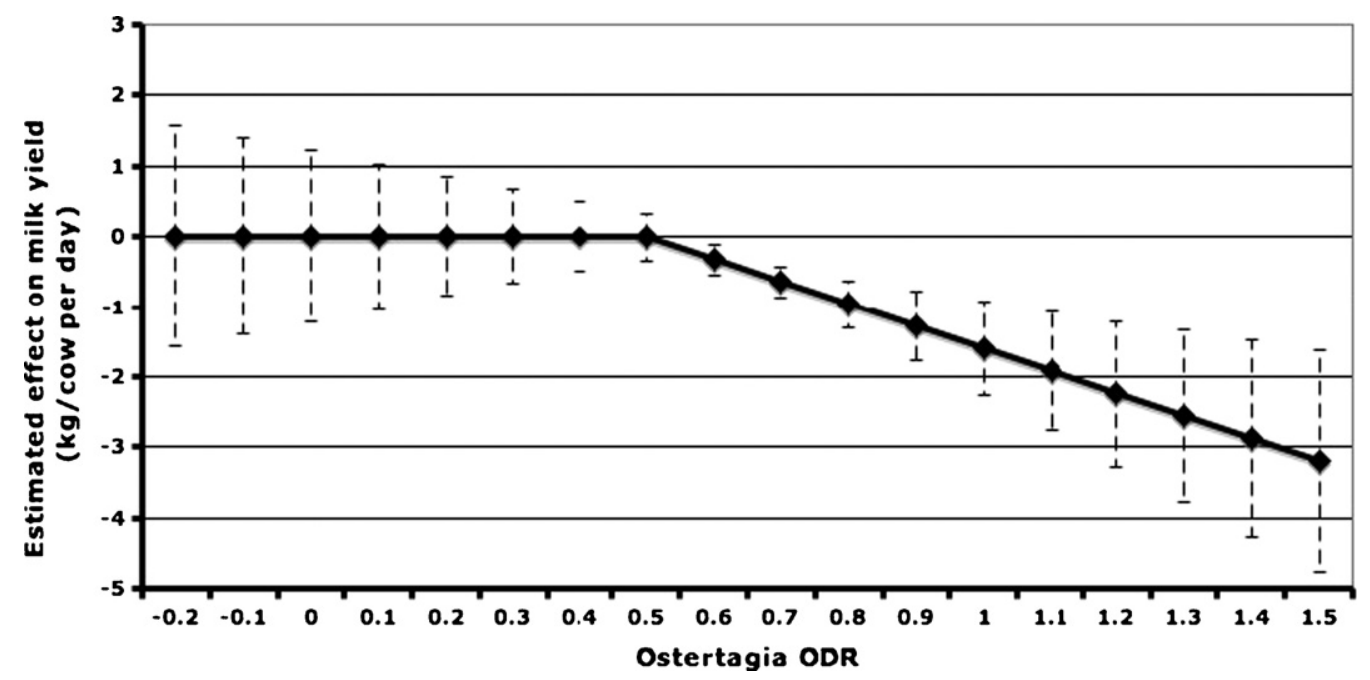

Fig. 2. Guide to interpretation of Bulk Tank Milk O. ostertagi ELISA titres (ODR) in relation to potential impact on individual daily milk yield in dairy herds.

to be considered along with the value of any additional potential benefits of treatment such as increased appetite, improved liveweight, condition score and reproductive performance (Forbes et al., 2004; Sanchez et al., 2002b). Other considerations include whether to treat the whole herd synchronously or each cow individually and whether to treat at housing, at calving or at some other time. Regardless of these considerations, when diagnostic values appropriate for different production situations and environments have been further validated, the test will provide an objective, quantitative assessment of a dairy herd's status, with respect to $O$. ostertagi antibodies, and thus furnish veterinarians, advisors and dairy farmers with a valuable decision-making tool.

\section{Acknowledgements}

Thanks are due to the many people, including Merial staff, local veterinarians animal health groups and milk recorders in the different countries who co-ordinated and assisted in the collection of the samples and transferring them to the laboratory in Ghent, where thanks are due to Lonneke Verbeek who performed the milk analyses. Particular thanks are also due to Dr. Harm Ploeger from the University of Utrecht and Dr. Flemming Skjøth of the Danish Cattle Federation for additional statistical analyses of the Dutch and Danish data, respectively.

\section{References}

Charlier, J., Claerebout, E., De Muelenaere, E., Vercruysse, J., 2005a. Associations between dairy herd management factors and bulk tank milk antibody levels against Ostertagia ostertagi. Veterinary Parasitology 133, 91-100.

Charlier, J., Claerebout, E., Duchateau, L., Vercruysse, J., 2005b. A survey to determine relationships between bulk tank milk antibodies against Ostertagia ostertagi and milk production parameters. Veterinary Parasitology 129, 67-75.

Charlier, J., Duchateau, L., Claerebout, E., Vercruysse, J., 2005c. Assessment of the repeatability of a milk Ostertagia ostertagi ELISA and effects of sample preparation. Preventive Veterinary Medicine 68, 277-288.

Charlier, J., Camuset, P., Claerebout, E., Courtay, B., Vercruysse, J., 2007a. A longitudinal survey of anti-Ostertagia ostertagi antibody levels in individual and bulk tank milk in two dairy herds in Normandy. Research in Veterinary Science 83, 194-197.

Charlier, J., Duchateau, L., Claerebout, E., Vercruysse, J., $2007 \mathrm{~b}$. Predicting milk-production responses after an autumn treatment of pastured dairy herds with eprinomectin. Veterinary Parasitology $143,322-328$.

Eysker, M., Ploeger, H.W., 2000. Value of present diagnostic methods for gastrointestinal nematode infections in ruminants. Parasitology 120 (Suppl), S109-119.

Eysker, M., van Aarle, D., Kooyman, F.N., Nijzink, A.M., Orsel, K., Ploeger, H.W., 2002. Exposure of dairy cows to nematode infections at the end of the grazing season in The Netherlands. Veterinary Parasitology 110, 93-100.

Forbes, A.B., Huckle, C.A., Gibb, M.J., 2004. Impact of eprinomectin on grazing behaviour and performance in dairy cattle with sub-clinical gastrointestinal nematode infections under continuous stocking management. Veterinary Parasitology $125,353-364$.

Fox, M.T., Hutchinson, M., Riddle, A., Forbes, A.B., 2007. Epidemiology of subclinical dairy cow nematode infections on five farms in England in 2002 and a comparison with results from 1978 to 1979. Veterinary Parasitology 146, 294-301.

Gross, S.J., Ryan, W.G., Ploeger, H.W., 1999. Anthelmintic treatment of dairy cows and its effect on milk production. The Veterinary Record 144, 581-587.

Guiot, A.-L., Charlier, J., Pravieux, J.-J., Courtay, B., Vercruysse, J., 2007. Relation entre la measure d' anticorps anti-Ostertagia sur lait 
de mélange et les paramètres de production laitière en France. Bulletin des GTV 38, 89-93.

Guitian, F.J., Dohoo, I.R., Markham, R.J., Conboy, G., Keefe, G.P., 2000. Relationships between bulk-tank antibodies to Ostertagia ostertagi and herd-management practices and measures of milk production in Nova Scotia dairy herds. Preventive Veterinary Medicine 47, 79-89.

Kloosterman, A., Verhoeff, J., Ploeger, H.W., Lam, T.J.G.M., 1993. Antibodies against nematodes in serum, milk and bulk milk samples as possible estimators of infection in dairy cows. Veterinary Parasitology 47, 267-278.

Kloosterman, A., Ploeger, H.W., Pieke, E.J., Lam, T.J.G.M., Verhoeff, J., 1996. The value of bulk milk ELISA Ostertagia antibody titres as indicators of milk production response to anthelmintic treatment in the dry period. Veterinary Parasitology 64, 197-205.

MAFF, 1980, Booklet 2154: Grazing plans for the control of stomach and intestinal worms in sheep and in cattle. Ministry of Agricultural, Fisheries and Food, 17 p.

Michel, J.F., Richards, M., Altman, J.F., Mulholland, J.R., Gould, C.M., Armour, J., 1982. Effect of anthelmintic treatment on the milk yield of dairy cows in England Scotland and Wales. The Veterinary Record 111, 546-550.

O'Farrell, K.J., Downey, N.E., Sherrington, J., 1986. The effect of anthelmintic treatment at calving on the subsequent milk production characteristics of dairy cows. Irish Veterinary Journal 40, 116-123.

Ploeger, H.W., Schoenmaker, G.J.W., Kloosterman, A., Borgsteede, F.H., 1989. Effect of anthelmintic treatment of dairy cattle on milk production related to some parameters estimating nematode infection. Veterinary Parasitology 34, 239-253.

Ploeger, H.W., Kloosterman, A., Bargeman, G., Von Wuijckhuise, L., Van den Brink, R., 1990. Milk yield increase after anthelmintic treatment of dairy cattle related to some parameters estimating helminth infection. Veterinary Parasitology 35, 103-116.

Sanchez, J., Dohoo, I., 2002. A bulk tank milk survey of Ostertagia ostertagi antibodies in dairy herds in Prince Edward Island and their relationship with herd management factors and milk yield. The Canadian Veterinary Journal 43, 454-459.

Sanchez, J., Dohoo, I., Nodtvedt, A., Keefe, G., Markham, F., Leslie, K., DesCoteaux, L., Campbell, J., 2002a. A longitudinal study of gastrointestinal parasites in Canadian dairy farms. The value of an indirect Ostertagia ostertagi ELISA as a monitoring tool. Veterinary Parasitology 107, 209-226.

Sanchez, J., Nodtvedt, A., Dohoo, I., DesCoteaux, L., 2002b. The effect of eprinomectin treatment at calving on reproduction parameters in adult dairy cows in Canada. Preventive Veterinary Medicine 56, 165-177.

Sanchez, J., Dohoo, I., Carrier, J., DesCoteaux, L., 2004a. A metaanalysis of the milk-production response after anthelmintic treatment in naturally infected adult dairy cows. Preventive Veterinary Medicine 63, 237-256.

Sanchez, J., Markham, F., Dohoo, I., Sheppard, J., Keefe, G., Leslie, K., 2004b. Milk antibodies against Ostertagia ostertagi: relationships with milk IgG and production parameters in lactating dairy cattle. Veterinary Parasitology 120, 319-330.

Sanchez, J., Dohoo, I., Leslie, K., Keefe, G., Markham, F., Sithole, F., 2005. The use of an indirect Ostertagia ostertagi ELISA to predict milk production response after anthelmintic treatment in confined and semi-confined dairy herds. Veterinary Parasitology $130,115-124$.

Sithole, F., Dohoo, I., Leslie, K., DesCoteaux, L., Godden, S., Campbell, J., Stryhn, H., Sanchez, J., 2005. Effect of eprinomectin treatment at calving on milk production in dairy herds with limited outdoor exposure. Journal of Dairy Science 88, 929-937.

Vercruysse, J., Claerebout, E., 2001. Treatment vs non-treatment of helminth infections in cattle: defining the threshold. Veterinary Parasitology 98, 195-214.

Yazwinski, T.A., Tucker, C.A., 2006. A sampling of factors relative to the epidemiology of gastrointestinal nematode parasites of cattle in the United States. Veterinary Clinics of North America: Food Animal Practice 22, 501-527. 